\title{
Autogenous Demineralized Dentin Graft for Third Molar Socket Regeneration - A Case Report
}

\section{Md. Arafat Kabir ${ }^{1 *}$, Masaru Murata ${ }^{2}$, Koaru Kusano², Toshiyuki Akazawa ${ }^{3}$ and Takanori Shibata ${ }^{1}$}

${ }^{1}$ Department of Reconstructive Surgery for Oral and Maxillofacial Region, School of Dentistry, Health Sciences University of Hokkaido, Hokkaido, Japan ${ }^{2}$ Department of Oral and Maxillofacial Surgery, School of Dentistry, Health Sciences University of Hokkaido, Hokkaido, Japan

${ }^{3}$ Industrial Research Institute, Hokkaido Research Organization, Hokkaido, Japan

\begin{abstract}
The purpose of this case report is to evaluate the effects of a patient's own demineralized dentin matrix (DDM) for socket preservation after extraction. A 27 year old male presented with slightly mesially inclined upper impacted right third molar with pericoronitis. Extraction of the impacted tooth was performed atraumatically and DDM granules were prepared from the extracted tooth. Firstly, the tooth was crushed by a newly developed auto-crush mill at 12,000 rpm for 60 seconds. The crushed granules were demineralized completely in $0.34 \mathrm{~N}$ nitric acid for 20 minutes and rinsed in distilled water. Immediate autograft of DDM was done into the tooth socket after 20 perforations into the socket surface. The results were evaluated clinically and radiographically at 3 and 12 months postoperatively. Dental X-ray just after the graft revealed radiopaque particles covering fully inside the socket. At 3 and 12 months of the follow-up, the socket appeared to be filled with uniform radiodense bone like tissue. The micro-CT and 3D micro-CT images at 12 months showed complete bone regeneration with no change in the vertical and horizontal dimension of the alveolar ridge. No significant difference was existed in radiodensity between new bone inside the socket and surrounding alveolar bone. The results of this case report suggest that autogenous DDM graft might be very effective as bone-forming materials for bone regeneration in the extracted socket.
\end{abstract}

Keywords: Demineralized dentin matrix; Graft; Tooth socket; Bone regeneration

\section{Introduction}

The management of the osseous defects distal to the second molars as a result of the surgical removal of impacted wisdom teeth can be a challenge. The reasons for third molar extraction are varied like pericoronitis, decay, risk of damage to adjacent teeth, cyst or tumor formation, or to facilitate orthodontic treatment [1]. The postextraction bone loss, a physiological phenomenon will take place with alveolar resorption and the subsequent formation of bone within the socket follows osteoblastic differentiation of osteoprogenitor cells [24]. This phenomenon continues for a period of weeks [4]. However, studies have documented that the bone volume following extractions decreased by $50 \%$ within 12 months, and two-thirds of this resorption took place during the first 3 months after extraction [5,6]. Bone resorption will result in a loss of socket width three-dimensionally that subsequently hamper the native alveolar ridge contour [2,3]. Therefore, maintaining 3-dimensional alveolar bone volume is required for ideal esthetic and functional restorations.

Bone grafting to augment skeletal healing has become one of the most common surgical techniques in recent years. Studies have shown that bone augmentation procedures with the use of graft materials may prevent progressive bone resorption [7,8]. Autogenous bone graft is considered the gold standard in regenerative procedures because of its osteoinduction, osteoconduction and osseointegration properties required for bone regeneration [9,10]. Despite these essential properties drawbacks involving autogenous bone grafting include need for the second surgery, donor site morbidity and limited availability have led to the challenging study for alternative biomaterials with osteoinductive potential $[11,12]$.

Healthy non-functional teeth extracted from human are considered as infective dental waste globally. High proportion of extracted sockets left untreated for physiological healing all over the world. However, it has been reported inadequate or failure of bone healing on the socket due to absence of bone graft material [6]. Human tooth is a rich source of stem cells, matrix, trace metal ions, and growth factors [13]. Although the tissue structures of bone and dentin are different, the ratio of components is similar (mineral $70 \%$, collagen $20 \%$, body fluid $10 \%$ by weight). After demineralization, dentin matrix is mainly composed of predominantly type-I collagen $(95 \%)$ and non-collagenous proteins including growth factors [13]. Growth factors identified in human dentin included insulin like growth factor I (IGF-I), skeletal growth factor/insulin like growth factor II (IGF-II), and transforming growth factor- $\beta$ (TGF- $\beta$ ) [14]. Considering these reports, human tooth-derived demineralized dentin matrix (DDM) could be defined as acid-insoluble collagen with bone inducing molecules.

The history of a bone-inducing research in dentin began with a report in 1967 that animal derived DDM induced bone formation in the intramuscular pockets [15]. Until now, several dentin studies have reported the osteoinductive potency of DDM and presence of BMP molecule in dentin matrix [16-20]. In addition, it was noted that DDM derived from human teeth, induced bone and cartilage independently in subcutaneous tissues of nude mice at 4 weeks after implantation [20,21]. As human tooth-derived DDM has been recycled as osteoinductive materials for local bone regeneration [20], therefore, this case report described the efficacy of autogenous DDM for bone regeneration in the extracted socket.

The aim of this clinical report was to evaluate the feasibility of the autogenous DDM on new bone formation during the healing of the extracted socket after a period of 12 months. The observation of

${ }^{*}$ Corresponding author: Md. Arafat Kabir BDS, PhD., Assistant Professo (Adjunct), Department of Reconstructive Surgery for Oral and Maxillofacial Region School of Dentistry, Health Sciences University of Hokkaido, 1757 Kanazawa, Tobestsu-cho, Ishikarigun, Hokkaido 061-0293, Japan, Tel: +81133232951; Fax: +81133231429; E-mail: kabir@hoku-iryo-u.ac.jp

Received October 14, 2015; Accepted October 27, 2015; Published November 01, 2015

Citation: Kabir MA, Murata M, Kusano K, Akazawa T, Shibata T (2015) Autogenous Demineralized Dentin Graft for Third Molar Socket Regeneration - A Case Report Dentistry 5: 343. doi:10.4172/2161-1122.1000343

Copyright: $\odot 2015$ Kabir MA, et al. This is an open-access article distributed under the terms of the Creative Commons Attribution License, which permits unrestricted use, distribution, and reproduction in any medium, provided the original author and source are credited. 
the new bone formation on the socket was evaluated clinically and radiologically.

\section{Case Report}

A 27 year old healthy non-smoker male with good oral hygiene required the extraction of upper right third molar as he complained of inability to sustain good oral care at the posterior aspect of the upper right jaw. The chief complains were food impaction and subsequent malodor. The patient had no remarkable medical history. Clinical examination revealed partially erupted third molar, mild inflammation in the pericoronal tissues and surrounding mucosal erythema. There was impacted food debris along with slight pain during pressing over the soft tissue covering the impacted tooth. Radiographic examination showed the impacted tooth was slightly buccally placed to the second molar. On the basis of the clinical and radiographic reports, the partially erupted third molar had to be extracted as it has led to pericoronitis in the posterior right maxillary region. Case situation was explained to the patient that impacted tooth should be removed but there will be susceptibility of pocketing formation at the distal to the second molar after extraction due to bone defect. The alveolar socket preservation protocol by using DDM was explained briefly to the patient and written informed consent was obtained from the patient after the risks and benefits were explained to him. This clinical case was approved by the Institutional Ethics Committee.

\section{Surgical procedure}

After administration of the local anesthesia with $2 \%$ mepivacaine containing epinephrine 1:100,000, an intrasulcular incision was made to raise a dental papilla and marginal gingiva. This exposed the marginal bone to allow visualization and measurement of the alveolar bone level. Extraction of the impacted tooth was performed atraumatically using a straight elevator and forceps. Inspection of the socket followed by debridement and curettage of the granulation tissue was done with saline solution. Immediate after extraction, patients own DDM was prepared. At first, the extracted tooth was crashed by a newly developed auto-crush mill (Osteo-Mill, Tokyo Iken Co.Ltd, Tokyo, Japan) at 12,000 rpm for 60 seconds with 15 saline-ice blocks. The crushed granules were demineralized in $0.34 \mathrm{~N}$ nitric acid $\left(\mathrm{HNO}_{3}\right)$ solution ( $\mathrm{pH} \mathrm{1.0)}$ for 20 minutes and extensively rinsed in distilled water (DW) to prepare patients own DDM (granule size 0.5-2.0 mm) (Figure 1). Just before the grafting of the DDM, 20 perforations by small round bur $(0.5 \mathrm{~mm}$ in diameter) were introduced on the socket surface. This strategy was performed to supply marrow-derived stromal cells and blood components for an initial acceleration of osteogenesis [22]. The autogenous DDM granules were inserted inside the socket incrementally with light condensation to allow blood vessels to grow in between the graft particles. Good wettability of the DDM with blood was observed just after the graft (Figure 2). The volume of the DDM was adequate to cover the whole socket. No barrier membrane was used to cover the graft and socket. The mesiobuccal and attached gingiva at the extraction site were stabilized with single interrupted suture. An immediate post-operative dental X-ray was obtained and the patient was prescribed a course of medication with post-operative instructions for 7 days, at which point the suture was removed. After 3 and 12 months, the patient was recall for follow-up examination and periapical X-ray and micro-CT was carried out to evaluate bone regeneration in the socket. Furthermore, tissue density was measured between the new bone and surrounding alveolar bone by Hounsfield Scale. Regions of interest (ROI) were $0.020 \mathrm{~cm}^{2}(\mathrm{n}=6)$ and the statistical analysis was performed by Student's t-test. The statistical significance was set at $\mathrm{p}<0.05$.
The healing of the post-extraction wound was uneventful and satisfactory by the qualitative clinical and radiographic assessments at regular intervals. Clinically, the gingival heights and widths appeared to maintain with the respective preoperative dimensions. At 7 days postoperatively, no signs of microbial infection, exudation, or dehiscence of the wound were observed. Periapical X-ray just after DDM graft revealed radiopaque particles covering fully inside the socket including a sharp radiopaque line of lamina dura (Figure $3 \mathrm{~A}$ and $3 \mathrm{~B})$. At 3 and 12 months of the follow-up, the alveolar socket appeared to be filled with uniform radiodense bone like tissue indicated that the socket healed fully with new bone (Figure 3C and 3D). In addition, lamina dura of the socket disappeared completely by bone remodeling. Gradual absorption of the DDM granules was observed on the sequential radiographic findings of the socket (Figure 3). At 12 months, micro-CT showed good trabecular bone regeneration throughout the socket. Autogenous DDM was absorbed completely and remodeled into new bone. There was no residue of DDM remnants on the graft site (Figure 4). No significant (NS) difference was existed in radiographic density between the alveolar bone and new bone. The Hounsfield Unit (HU) values at newly formed bone and alveolar bone were $935.5 \pm$ 22.0 and $796.0 \pm 43.3(n=6$; NS) respectively (Figure 5). Finally, the 3D micro-CT revealed no change in the vertical and horizontal dimension of the alveolar ridge (Figure 6).

\section{Discussion}

This case report demonstrated clinical and radiographic observations on post-extraction healing of the third molar socket that

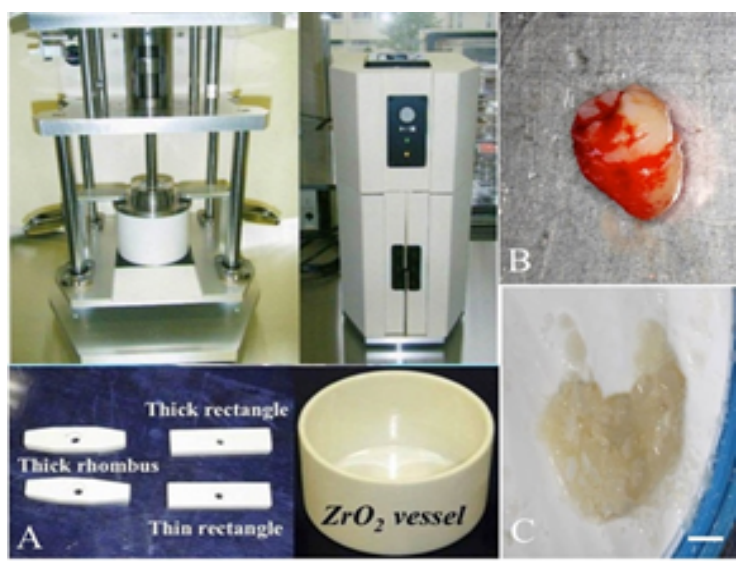

A. Auto-crush mill with $\mathrm{ZrO}_{2}$ vessel and blade; B. Extracted third molar; C. DDM prepared from extracted tooth (Bar $=1 \mathrm{~mm})$

Figure 1: Preparation of DDM

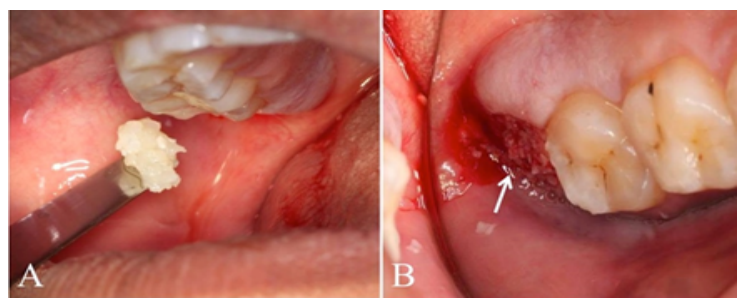

A. DDM installation into the extracted socket (White DDM particles); B. Just after DDM graft, Wettability of DDM with blood (white arrow).

Figure 2: Installation of DDM 


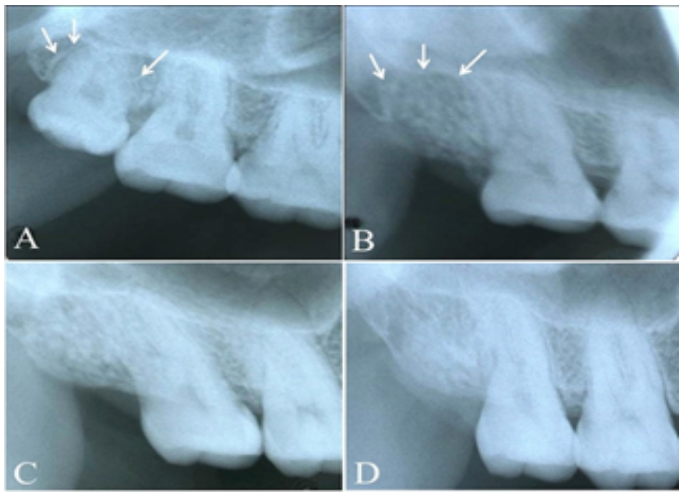

A. Preoperative periapical X-ray (Arrows indicate lamina dura); B. Immediate postoperative radiograph showing socket filled with radiopaque DDM particles; C. After 3 months, radiograph showing higher density with DDM remnants and unclear lamina dura; D. At 12 months, complete bone formation with native bone trabecular structure and density similar to the surrounding alveolar bone, complete disappearance of lamina dura indicating successful bone healing.

Figure 3: Sequential radiological findings.
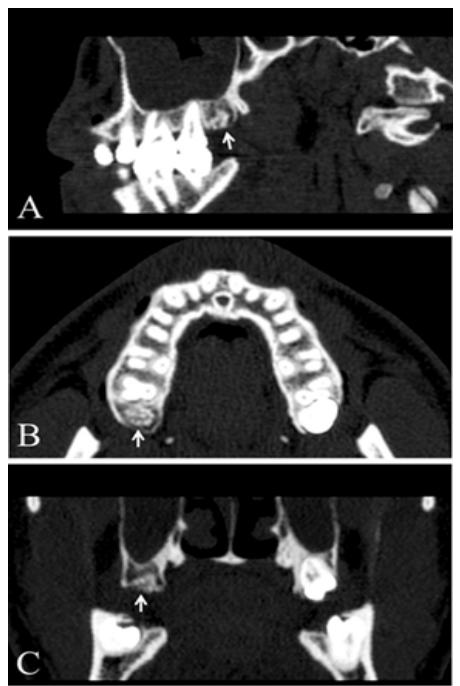

Sagittal (A), axial (B) and longitudinal (C) view of the CT scans showing new bone formation (arrow) into the interior of extraction socket.

Figure 4: The micro-CT images at 12 months follow-up visit.

was treated with patients own DDM. Our concern in the case report is to elucidate the healing potential of the grafted socket with autogenous DDM.

Extensive studies have been conducted to evaluate biocompatibility, osteoinductivity, and osteoconductive properties of DDM [13,15$17,23]$. The chemotactic properties of the DDM for osteoprogenitor cells and osteoblasts have been verified for the promotion of the bone formation [24]. The effectiveness of the DDM on the extracted socket was tested wherein the bone radiopacity with enhanced healing process was observed $[25,26]$. Periods of clinical and radiographic observations focusing on the changes in radiopacity between the graft material and bone confirmed that the DDM was biocompatible and clinically easy to use. Therefore, the use of autogenous DDM in this case is thought to provide structural components similar to that of human bone and with its osteoinductive and conductive capability, DDM served as a scaffold for bone formation [13].

In this case report, DDM was used as a collagenous biomaterial including growth factors for bone regeneration [13]. The patient received DDM graft without any complication and achieved good clinical outcomes. The gingiva and mucosa healed very well over the socket with no associated infections. The DDM was placed in the extraction socket with the aim to preserve the bone quality, to improve both vertical and horizontal bone dimensions, and to promote soft tissue healing. By using the patient's own DDM to regenerate bone, we avoided any possible immune or biocompatibility problems. The grafted DDM was followed by radiographic observations. The biomaterial was readily identifiable on X-ray, being much denser than the adjacent bone. This characteristic is very useful for the radiographic follow-up of the socket healing, because the radiopacity changes as the material is resorbed and replaced by new bone. Radiographic evaluation showed noticeable enhancement of radiopacity on the grafted socket by the autogenous DDM. Complete bone healing was noted in the socket. When the socket was filled with DDM, a sharp radiopaque layer of lamina dura was observed just after graft but it disappeared after 3 months. The quantity of radiopaque particles was less visible in sequential radiological images. With increasing time, autogenous DDM was absorbed gradually and remodeled into new bone. The micro-CT images revealed no DDM remnants and remodeled bone with trabecular structure inside the socket at 12 months. No significant difference was existed between the density of new bone inside the socket and the adjacent alveolar bone. The results

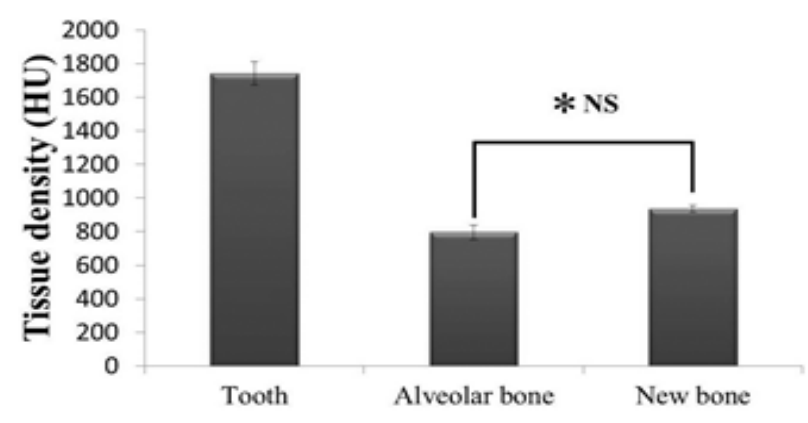

Hard tissues

Bone density was measured at tooth, surrounding alveolar bone and new bone inside the socket. With ongoing graft healing, no significant different observed between the $\mathrm{HU}$ of new bone and native alveolar bone at 12 months postoperative follow-up. Regions of interest (ROI) were $0.020 \mathrm{~cm}^{2}$; $n=6$. The values are given as mean $\pm S D$. *NS $=$ no significant.

Figure 5: Bone density in Hounsfield units (HU).
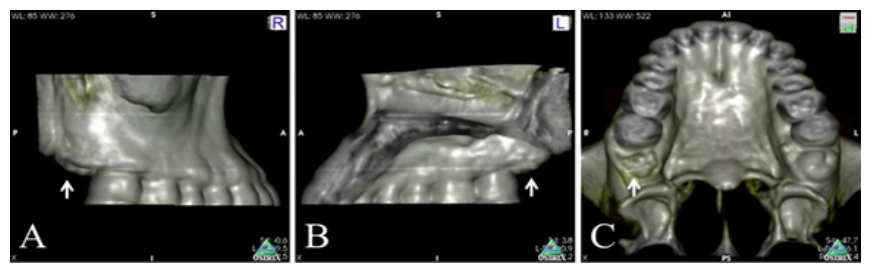

Buccal $(A)$, lingual $(B)$ and occlusal $(C)$ view of the $3 D$ micro-CT showing no change in vertical and horizontal dimension of the alveolar ridge at 12 months postoperative follow-up. Arrow indicates graft site.

Figure 6: 3D micro-CT images at 12 months follow-up visit. 
of the HU value indicated that the new bone was remodeled completely by DDM. Eventually, DDM contributed to the vertical and horizontal dimension of the alveolar ridge evaluated by $3 \mathrm{D}$ micro-CT images.

Autogenous DDM graft can be regarded as an innovative material owing to its similar composition and properties like bone graft. Presence of growth factors, bio-compatibility, bioabsorption and low treatment cost enabled this material a realistic alternative over artificial biomaterials. Considering the results of this case report, this novel technology using human DDM should spread to the bone regenerative field for patients in near future.

\section{Conclusion}

The clinical and radiographic outcomes of this case were satisfactory, with no associated complications. The autogenous DDM seems to be a biomaterial capable of achieving preservation of the alveolar bone when it is positioned immediately in post-extraction socket by facilitating the formation of new bone at 12 months after graft. This resorbable material allows predictable and reproducible bone regeneration. As advantages, it can be noted its low morbidity, easy handling and great radiopacity, allowing radiographic follow-up of the area. We concluded that autogenous DDM graft might be very useful as bone-forming materials for bone regeneration in the extracted socket.

\section{Acknowledgments}

The authors would like to acknowledge the help of Dr. Masaru Murata for the surgical procedure.

\section{References}

1. Peterson LJ (1998) Principles of management of impacted teeth. In: Contemporary Oral and Maxillofacial surgery. ( $3^{\text {rd }}$ edn), Mosby, Saint Loius.

2. Atwood DA, Coy WA (1971) Clinical, cephalometric and densitometric study of reduction of residual ridges. J Prosthet Dent 26: 280-295.

3. Watt DM, Likeman PR (1974) Morphological changes in the denture bearing area following the extraction of maxillary teeth. Br Dent J 136: 225-235.

4. Guglielmotti MB, Cabrini RL (1985) Alveolar wound healing and ridge remodeling after tooth extraction in the rat: a histologic, radiographic, and histometric study. J Oral Maxillofac Surg 43: 359-366.

5. Schropp L, Wenzel A, Kostopoulos L, Karring T (2003) Bone healing and soft tissue contour changes following single-tooth extraction: a clinical and radiographic 12-month prospective study. Int J Periodontics Restorative Dent 23: 313-323.

6. Van der Weijden F, Dell'Acqua F, Slot DE (2009) Alveolar bone dimensional changes of post-extraction sockets in humans: a systematic review. J Clin Periodontol 36: 1048-1058.

7. Bernstein S, Cooke J, Fotek $\mathrm{P}$, Wang HL (2006) Vertical bone augmentation: Where are we now? Implant Dent 15: 219-228.

8. Scheyer ET, Schupbach P, McGuire MK (2012) A histologic and clinical evaluation of ridge preservation following grafting with demineralized bone matrix, cancellous bone chips, and resorbable extracellular matrix membrane. Int J Periodontics Restorative Dent 32: 543-552.

9. Bauer TW, Muschler GF (2003) Bone graft materials. An overview of the basic sciences. Clin Orthop Relat Res 371:10-27.

10. Rosenberg E, Rose LF (1998) Biologic and clinical considerations for autografts in periodontal regeneration therapy. Dent Clin North Am 42: 467-490.
11. Younger EM, Chapman MW (1989) Morbidity at bone graft donor sites. J Orthop Trauma 3:192-195.

12. Carlsen A, Gorst-Rasmussen A, Jensen T (2013) Donor site morbidity associated with autogenous bone harvesting from the ascending mandibular ramus. Implant Dent 22: 503-506.

13. Murata M, Akazawa T, Mitsugi M, Kabir MA, UM IW, et al. (2013) Autograft of dentin materials for bone regeneration. In: Pignatello R (Ed.), Advances in Biomaterials Sciences and Biomedical Applications. InTech, Croatia.

14. Finkelman RD, Mohan S, Jennings JC, Taylor AK, Jepsen S, et al. (1990) Quantitation of growth factors IGF-I, SGF/IGF-II, and TGF-beta in human dentin. J Bone Miner Res 5: 717-723.

15. Yeomans JD, Urist MR (1967) Bone induction by decalcified dentin implanted into oral, osseous and muscle tissues. Arch Oral Biol 12: 999-1008.

16. Bang G, Urist MR (1967) Bone induction in excavation chambers of decalcified dentin. Arch Surg 94: 781-789.

17. Huggins CB, Urist MR (1970) Transplantation: Rapid induction of alkaline phosphatase and cartilage. Science 167: 896-897.

18. Inoue T, Deporter DA, Melcher AH (1986) Induction of chondrogenesis in muscle, skin, bone marrow, and periodontal ligament by demineralized dentin and bone matrix in vivo and in vitro. J Dent Res 65: 12-22.

19. Besso K, Tanaka N, Matsumoto J, Tagawa T, Murata M (1991) Human dentinmatrix-derived bone morphogenetic protein. J Dent Res 70: 171-175.

20. Murata M, Kawai T, Kawakami T, Akazawa T, Tazaki J, et al. (2010) Human acid-insoluble dentin with BMP-2 accelerates bone induction in subcutaneous and intramascular tissues. J Ceram Soc Jpn 118: 438-441.

21. Murata M, Sato D, Akazawa T, Taira T, Sasaki T, et al. (2003) Bone and cartilage induction in nude mice by human demineralized dentin matrix. J Hard Tissue Biol 11: 110-114.

22. Murata M, Huang BZ, Shibata T, Imai S, Nagai N, et al. (1999) Bone augmentation by recombinant human BMP-2 and collagen on adult rat parietal bone. Int J Oral Maxillofac Surg 28: 232-237.

23. Murata M, Sato D, Hino J, Akazawa T, Tazaki J, et al. (2012) Acid-insoluble human dentin as carrier material for recombinant human BMP-2. J Biomed Mater Res 100: 571-577.

24. Gomes MF, Banzi EC, Destro MF, Lavinicki V, Goulart Md (2007) Homogenous demineralized dentin matrix for application in cranioplasty of rabbits with alloxan-induced diabetes: histomorphometric analysis. Int J Oral Maxillofac Implants 22: 939-947.

25. Kabir MA, Murata M, Kusano K, Zakaria MS, Noor AHM, et al. (2014) Radiological evaluation of human dentin autografts in Bangladesh. $\mathrm{J}$ Hard Tissue Biol 23: 363-370.

26. Kim YK, Kim SG, Byeon JH, Lee HJ, Um IU, et al. (2010) Development of a novel bone grafting material using autogenous teeth. Oral Surg Oral Med Oral Pathol Oral Radiol Endod 109: 496-503. 